\title{
KINETICS AND MECHANISM FOR THE CONFORMATIONAL TRANSITION IN p-GUANIDINOBENZOATE BOVINE TRYPSINOGEN INDUCED BY THE ISOLEUCINE-VALINE DIPEPTIDE
}

\author{
H.J. NULIE and E. NEUMANN \\ Max-Planck-Institut für Biochemie, D-8033 Martinsried bei München, W-Germany
}

Received 10 April 1979

Revised manuscript received 20 June 1979

\begin{abstract}
The interaction between p-guanidinobenzoate-trypsinogen and the isoleucine-valine dipeptide has been investigated by temperature-jump relaxation spectrometry. Using the absorbance at $281 \mathrm{~nm}$ the concentration dependence of the relaxation purameters is consistent with the conventional induced-fit model: rapid ligand binding coupled to a slower intramolecular change; some alternative mechanisms can be excluded. At $296 \mathrm{~K}, 0.1 \mathrm{M}$ Tris $\mathrm{HCl}, \mathrm{pH}=7.4$, the dissociation equilibrium constant for the overall process is $\bar{K}-5.1(\neq 0.2) \times 10^{-5} \mathrm{M}$; for the binding step $K_{1}=2.3( \pm 0.3) \times 10^{-3} \mathrm{M}$ and the rate constants for the structural change are $k_{2}=26( \pm 6) \mathrm{s}^{-1}$ and $k_{-2}=0.61( \pm 0.04) \mathrm{s}^{-1}$; the overall dissociation reaction enthalpy is $\Delta H^{0}=$ $26( \pm 6) \mathrm{kJmol}^{-1}$ and the reaction entropy is $\Delta S^{0}=4( \pm 20) \mathrm{kJ}^{-1} \mathrm{~mol}^{-1}$. In combination with CD and $X-r a y$ crystallographic data, the results of this study suggest that the binding of the dipeptide to a trypsinogen-like, partially disordered conformation induces a transition to a trypsin-like highly ordered structure.
\end{abstract}

\section{Introduction}

The regulation of enzymatic activity by limited proteolysis of zymogens is a well known biological control device; however, the mechanism of the conversion from the inactive form of the protein to the catalytically active species usually is not known in detail. In the case of bovine trypsinogen, $X$-ray crystallographic analysis has suggested that the formation of active trypsin is a transition of a partially disordered protein to a highly ordered structure [1]. This structural transition to the trypsin form can be achieved either by the intrusion of the isoleucine (Ile)-16-valine(Val)-17 N-terminus into the ne-16 binding cleft or by binding of the pancreatic trypsin inhibitor to trypsinogen.

If instead of the covalently bound $\mathrm{N}$-terminus the free dipeptide Ile-Val is offered to trypsinogen, no transition is observed. However, if the zymogen is mcdified by covalent attachment of the p-guanidinobenzoate (pGB) group, then the binding of free Ile-Val causes the formation of a trypsin-like structure from pGB-trypsinogen; this transition is characterized by a distinct change in the circular dichroism [2] .

Recently we have found that the binding of the di- peptide is accompanied by a small change in the optical absorption at $281 \mathrm{~nm}$. In particular, a chemical relaxation is observed in the same concentration range of pGB-trypsinugen and Ile-Val in which the circular dichroisin spectrum (CD) indicates the change from the partially disordered trypsinogen conformation to the highly ordered trypsin-like structure. Whereas the X-ray diffraction and CD-data allow the specification of the conformational states involved, a mechanistic discrimination between several pathways connecting these states is only possible by a kinetic analysis.

The relaxation kinetic data presented here provide evidence that the dipeptide binds predominantly to the partially disordered protein and subsequently induces a slower intramolecular change. The order of magnitude of the kinetic constants is suggestive of a major structural change induced by the binding of the dipeptide to the zymogen.

\section{Materials and methods}

The p-guanidinobenzoate-derivative of trypsinogen was prepared according to Kerr et al. [3] . Crystalline 
bovine trypsinogen was from Merck (Darmstadt), the acylating agent $\mathrm{p}$-nitrophenyl-p'-guanidinobenzoate and the Ile-Val dipeptide were a gift from Dr. W. Bode (Martinsried). The total protein concentration in the reaction solution was determined by a calibrated Biuretmethod; the fraction of pGB-trypsinogen was evaluated from the circular dichroism intensity observed at 269 nm [4]. In the experiments described here the degree of acylation was $67 \%$.

Temperature-jump perturbations of the interaction equilibrium between pGB-trypsinogen and the lle-Val dipeptide were applied to solutions of $0.1 \mathrm{M}$ Tris $\cdot \mathrm{HCl}-$ buffer at $\mathrm{pH}=7.4$. The Joule heating time of the solution in the observation cell was $9 \mu \mathrm{s}$; the corresponding temperature increase was from $293 \mathrm{~K}$ to $296 \mathrm{~K}$. Adjustment of the equilibrium to the raised temperature was monitored in the absorbance mode at $\lambda=281 \mathrm{~nm}$. The temperature-induced changes in the absorbance were very small; the relative change of the light intensity for a jump of $3 \mathrm{~K}$ amounted to a few tenths of a percent. A recent development (by C.R. Rabl) of a highresolution chemical relaxation spectromter [5] allows reliable recording of relaxation processes in the time range between $5 \mu$ s and several seconds.

Monitoring the interaction between pGB-trypsinogen ( $T$ ) and Ile-Val (I) in an equilibrium titration series by means of the circular dichroism of the reaction solution yielded a value of $\bar{K}=4.3 \times 10^{-5} \mathrm{M}$ for the overall equilibrium dissociation constant in $0.02 \mathrm{M}$ $\mathrm{CaCl}_{2}, 0.5 \mathrm{M}$ Tris $\cdot \mathrm{HCl}$ buffer, $\mathrm{pH}=7.5$, at $20^{\circ} \mathrm{C}[4]$. Therefore the relaxation kinetic titration experiments were performed in comparable concentration ranges. The total enzyme concentration was kept fixed at (a) $c_{\mathrm{T}}^{\mathbf{6}}=50 \mu \mathrm{M}$ and (b) $c_{\mathrm{T}}^{0}=20 \mu \mathrm{M}$ and the dipeptide concentration was varied between $60 \mu \mathrm{M}$ and $500 \mu \mathrm{M}$. The relaxation spectra were registered in a Bruker transient recorder and displayed on a Tektronix dual beam oscilloscope; simulated relaxation spectra from a calibrated multiple exponential function generator (C.R. Rabl, MPI für Biochemie, Martinsried) were superimposed to evaiuate relaxation times and amplitudes. Usually three to five temperature jump experiments at a given concentration of the reactants were sufficient to assure good experimental reproducibility. Under the applied experimental conditions, the acylated enzyme is slowly hydrolyzed, the half-time of this process being approximateiy one day [3]. Therefore, for each temperature jump experiment, fresh solutions were mixed

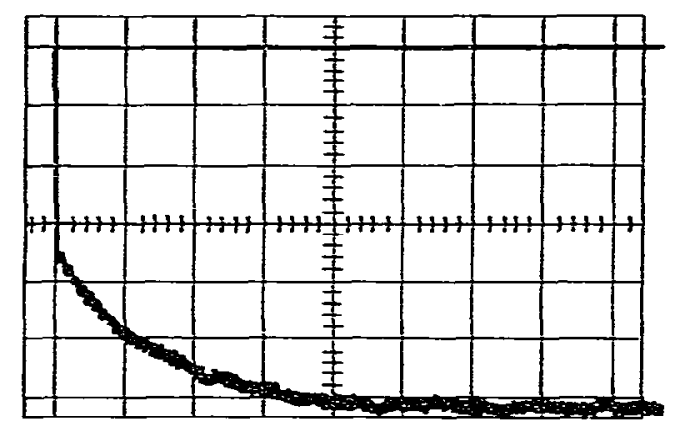

Fig. 1. Relaxation spectrum of Ile-Val (I) and pGB-trypsinogen (T) in $0.1 \mathrm{M} \mathrm{Tris} / \mathrm{HCl}$ buffer, at $\mathrm{pH}=7.4$ and $296 \mathrm{~K}$. The total signal before the temperature jump was adjusted to $20 \mathrm{~V} ; c_{\mathrm{T}}^{0}=$ $2 \times 10^{5} \mathrm{M}, c_{1}^{0}=3 \times 10^{-4} \mathrm{M} ;$ abscissa scale $0.2 \mathrm{~s} / \mathrm{div}$, ordinate scale $20 \mathrm{mV} / \mathrm{div}$.

to the desired concentrations; thus concentration uncertainties were reduced to a minimum, and a solution was processed not longer than $20 \mathrm{~min}$.

\section{Results}

The time range in which chemical relaxation processes can be reliably resolved under the given experimental conditions lies between the heating time $\left(\tau_{h}=\right.$ $9 \mu \mathrm{s})$ and several seconds, if cooling corrections are applied. In this time interval neither the enzyme nor the dipeptide alone show any chemical relaxation process observable in the absorbance mode. Only the mixture of protein and dipeptide exhibits a clear but biphasic relaxation; (fig. 1). The initial rapid absorbance decrease is associated with a concentration-independent time constant which is $\leqslant \tau_{h}$. This instantaneous change is attributed to the dilution of the reaction mixture as the temperature is increased [6] ; it agrees roughly with an estimation of the signal change calculated for the aqueous protein solutions using the corresponding values for the thermal expansion and the extinction coefficients, respectively.

The rapid signal is followed by a slower change in the absorbance. Under all experimental conditions, this slower absorbance decrease is a single exponential. Furthermore, this relaxation exhibits the characteristic features of a concentration-dependent chemical process. Fig. 2 shows that the observed relaxation times, mea- 




Fig. 2. Time constants $r$ of the slow relaxation mode versus the ratio of total concentrations of dipeptide (I) and protein (T) with theoretical fitting; (a) $c_{\mathrm{T}}^{\mathrm{o}}=5 \times 10^{-5} \mathrm{M}$, (b) $c_{\mathrm{T}}^{0}=2 \times 10^{-5}$ $M$; conditions as in fig. 1 .

sured at two constant enzyme concentrations, decrease from about $0.8 \mathrm{~s}$ to $0.2 \mathrm{~s}$ with increasing dipeptide concentrations. At higher ratios $c_{\mathbb{I}}^{0} / c_{\mathrm{T}}^{0}$ of the total concen-

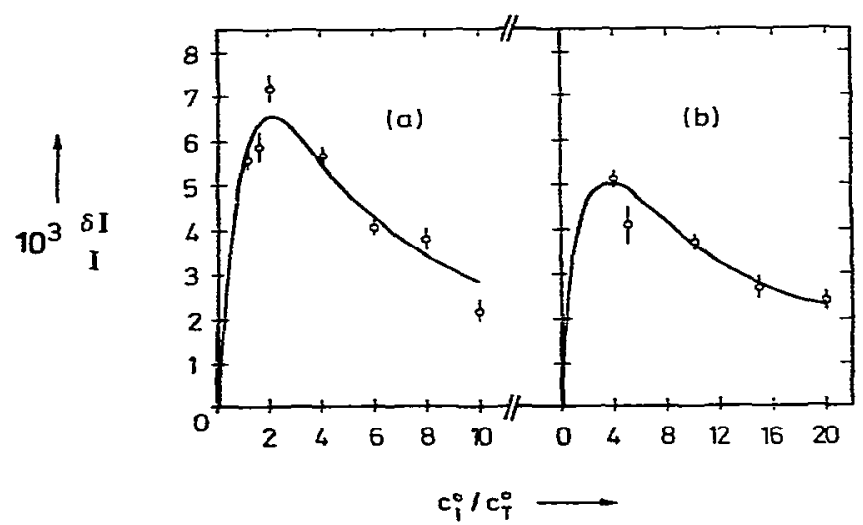

Fig. 3. Absorbance amplitudes of the siow relaxation mode versus the ratio of total concentrations of dipeptide and protein and theoretical fitting to eqs. (2) and (8); see text and table 1 ; (a) $c_{\mathrm{T}}^{\mathrm{O}}=5 \times 10^{-5} \mathrm{M} ;$ (b) $c_{\mathrm{T}}^{\mathrm{o}}=2 \times 10^{-5} \mathrm{M}$. trations of protein, $c_{\mathrm{T}}^{0}$, and dipeptide $c_{\mathrm{I}}^{0}$, respectively, the time, constants for both the lower and higher protein concentration tend to become independent of the dipeptide concentration. As seen in fig. 3 , the amplitudes of the relaxation signals pass through a maximum, which is located at $\left(c_{\mathrm{I}}^{0} / c_{\mathrm{T}}^{0}\right)_{\mathrm{m}}=1+\bar{K} / c_{\mathrm{T}}^{0}$ (see [6]), where $\bar{K}$ represents the overall dissociation equilibrium constant of the observed chemical process.

For the theoretical analysis, it is important that under all experimental conditions and over a wide range of dipeptide concentration, only one chemical relaxation is observed. Furthermore, the reactions underlying the experimental data depicted in figs. $2-4$, occur in the same concentration ranges and under similar experimental conditions where large changes in the CD-spectrum demonstrate that the ligand causes a structural change from the trypsinogen-like to a trypsin-like conformation $[2,4]$. As discussed below, the range of relaxation times between $0.2 \mathrm{~s}$ and $0.8 \mathrm{~s}$ suggests a more complex equilibrium process involving structural isomerizations rather than a simple bimolecular binding step. Therefore the equilibrium constant determined previously by a thermodynamic method [4] only represents an overall process probably consisting of several elementary steps.

\section{Relaxation kinetic analysis}

CD-data and X-ray diffraction analysis show that the free protein has the zymogen-like structure $(T)$, whereas the complex with the dipeptide (I) has a trypsin-like conformation $\left(T^{\prime}\right)$. In general however, the protein may equilibrate between the limit structures $T$ and $T^{\prime}$ and the dipeptide may separately associate with both conformational states. A minimum reaction model has therefore to incorporate a least two bimolecular elementary equilibria and two structural isomerization processes as represented in scheme 1 . The isomerizations may of course involve a whole series of individual structural transitions.

$$
\begin{aligned}
& \bar{I}+\mathrm{T} \underset{k_{-1}}{\stackrel{k_{1}}{\rightleftharpoons}} \mathrm{IT} \\
& k_{-3} \mid k_{3} \quad k_{-2} \| k_{2} \\
& \mathrm{I}+\mathrm{T}^{\prime} \stackrel{k_{4}}{\underset{k_{-4}}{\rightleftharpoons}} \mathrm{IT}^{\prime}
\end{aligned}
$$

Scheme 1 
Scheme 1 is characterized by three independent relaxation modes [7] ; this is reflected in the redundancy relation $K_{1} K_{2}=K_{3} K_{4}$. The overall equilibrium dissociation constant for this general scheme is given by

$\bar{K}=\frac{\bar{c}_{\mathrm{I}}\left(\bar{c}_{\mathrm{T}}+\bar{c}_{\mathrm{T}^{\prime}}\right)}{\bar{c}_{\mathrm{IT}}+\bar{c}_{\mathrm{IT}}}=K_{1} \frac{1+K_{3}}{1+K_{2}}$.

The bimolecular equilibrium constants $K_{1}$ and $K_{4}$ are defined as dissociation constants. The structural isomerizations are characterized by $K_{3}=\bar{c}_{\mathrm{T}} / \bar{c}_{\mathrm{T}^{\prime}}$ and $K_{2}=$ $\bar{c}_{\mathrm{IT}} / \bar{c}_{\mathrm{IT}}$ '. The bars denote equilibrium concentrations. The results of the present relaxation kinetic study demonstrate that only one reaction pathway in the general scheme is dominantly populated; otherwise we should have observed at least two relaxation processes during the kinetic titration over the large range of dipeptide concentrations, independent of which protein species is optically monitored. Another indication that one pathway is dominant is that no relaxation is observed with the free protein alone. Either there is no optical change connected with the transition $T \gtrless T^{\prime}$ or more probably, this equilibrium is shifted far to one side or the other. As judged from the X-ray diffraction and CD-data [4], we have to conclude that $\bar{c}_{\mathbf{T}} \gg \bar{c}_{T^{\prime}}$ i.e. the equilibrium concentration of the trypsin-like species $T^{\prime}$ is always very small. Therefore the relaxation amplitude associated with the $T \rightleftharpoons T^{\prime}$ equilibrium is also very small and may escape detection.

This preliminary inspection of the data suggests that the protein-ligand interaction may be described in terms of either of the limit pathways of the general scheme.

\subsection{Induced-fit model}

One of the limit pathways in scheme 1 represents the conventional induced-fit model [8], where a rapid bimolecular equilibrium is coupled to an intramolecular isomerization which is very often rate-limiting (scheme 2).

$\mathrm{I}+\mathrm{T} \frac{k_{1}}{k_{-1}} \mathrm{IT} \frac{k_{2}}{\vec{k}_{-2}} \mathrm{IT}^{\prime}$.

Scheme 2

Defining the elementary equilibrium constants as $K_{1}=$ $\bar{c}_{\mathrm{I}} \bar{c}_{\mathrm{T}} / \bar{c}_{\mathrm{IT}}=k_{-1} / k_{1}$ and $K_{2}=c_{\mathrm{IT}} / c_{\mathrm{IT}}{ }^{\prime}=k_{-2} / k_{2}$, respectively, the overall dissociation constant for the inducedfit model is then given by $\bar{K}=K_{1} /\left(1+K_{2}^{-1}\right)$.

\subsection{Selective binding model}

The other limit case for protein-ligand interactions may be called the pathway of selective binding. According to scheme 3 , the dipeptide can only bind to the trypsin-like form $T^{\prime}$, thereby shifting the concentrations of the two unbound protein states.

$$
\begin{gathered}
\mathbf{T} \\
k_{-3} \mid k_{3} \\
\mathbf{I}+\mathbf{T}^{\prime} \stackrel{k_{4}}{\stackrel{k_{-4}}{\rightleftharpoons}} \mathbf{I T}^{\prime}
\end{gathered}
$$

Scheme 3

With the definitions of $K_{3}=\bar{c}_{T} / \bar{c}_{T^{\prime}}=k_{-3} / k_{3}$ and of $K_{4}=\bar{c}_{\mathbf{I}} \bar{c}_{\mathrm{T}^{\prime}} / \bar{c}_{\mathrm{IT}^{\prime}}=k_{-4} / k_{4}$ the overali dissociation constant for the model of selective binding is $\bar{K}=\left(1+K_{3}\right) K_{4}$.

\subsection{Relaxation amplitudes}

In the limit of small changes, $\delta A$, in the absorbance, $A$, in response to a small temperature increas,$\delta T$, the relative change of transmitted light intensity $\delta I / I$ monitored on the oscilloscope screen is related to the temperature change $\delta T$ in the observation cell $[7,9]$. If the relative light intensity change is very small, such that $\delta I / I=-\delta A$, the relaxation amplitude is given by:

$\frac{\delta I}{I}=-\ln 10\left[\sum_{i=1}^{n} \nu_{i}\left(\frac{\partial A}{\partial c_{i}}\right)_{c \neq c_{i}}\right] \cdot \Gamma \frac{\Delta H^{0}}{R T^{2}} \delta T$.

In eq. (2), $\nu_{i}$ is the stoichiometric coefficient (negative for reactants and positive for products) of species $i$ (contributing to the total absorbance of $n$ species) the concentration of which changes by an amount $\delta c_{i} ; R$ is the gas constant, $T$ is the absolute temperature, $\Delta H^{0}$ is the molar reaction enthalpy for one stoichiometric transition, and the factor In 10 accounts for the decadic absorbance scale; $\Gamma=\left(\Sigma \nu_{i}^{2} / \bar{c}_{i}\right)^{-1}$ is the amplitude factor.

For the two limit pathways represented in schemes 2 and 3 , respectively, with rapid bimolecular steps, the slow relaxation reflects an overall process. Thus $\Delta H^{0}$ and $\Gamma$ are normal mode parameters characterizing the overall reaction. Under the specified conditions, the amplitude factor for the induced fit-model (scheme 2) 
is

$\Gamma=\left(\frac{1}{\bar{c}_{I}}+\frac{1}{\bar{c}_{\mathrm{T}}}+\frac{1}{\bar{c}_{\mathrm{IT}}+\bar{c}_{\mathrm{IT}}}\right)^{-1}$

and for the mociel of selective binding (scheme 3 ) we have

$\Gamma=\left(\frac{1}{\bar{c}_{\mathrm{I}}}+\frac{1}{\bar{c}_{\mathrm{T}}+\bar{c}_{\mathrm{T}^{\prime}}}+\frac{1}{\bar{c}_{\mathrm{IT}}}\right)^{-1}$.

For independent numerical analysis the amplitude factor $\Gamma$ should be written in terms of stoichiometric concentrations. Applying the law of mass action to the induced-fit model we obtain:

$$
\begin{aligned}
& 2\left(\bar{c}_{\mathrm{IT}}+\bar{c}_{\mathrm{IT}}\right)=\left(c_{\mathrm{I}}^{0}+c_{\mathrm{T}}^{0}+\bar{K}\right) \\
& \quad-\left[\left(c_{\mathrm{I}}^{0}+c_{\mathrm{I}}^{0}+\bar{K}\right)^{2}-4 c_{\mathrm{I}}^{0} c_{\mathrm{T}}^{0}\right]^{1 / 2}
\end{aligned}
$$

Inserting eq. (5) into the mass conservation relation

$\bar{c}_{\mathrm{I}}+\bar{c}_{\mathrm{T}}=c_{\mathrm{I}}^{0}+c_{\mathrm{T}}^{0}-2\left(\bar{c}_{\mathrm{IT}}+\bar{c}_{\mathrm{IT}^{\prime}}\right)$

we replace the free concentrations of the reactants by total concentrations and the overall equilibium constant $\bar{K}$ :

$\bar{c}_{\mathrm{I}}+\bar{c}_{\mathrm{T}}=-\bar{K}+\left[\left(c_{\mathrm{I}}^{0}+c_{\mathrm{T}}^{0}+\bar{K}\right)^{2}-4 c_{\mathrm{I}}^{0} c_{\mathrm{T}}^{0}\right]^{1 / 2}$.

For the model of selective binding, the corresponding substitution of free concentrations by stoichiometric concentrations is along similar lines. It can then be shown that both the induced-fit model and the model of selective binding are described by the same relationship

$\Gamma=\frac{\bar{K}}{2}\left(\frac{c_{\mathrm{I}}^{0}+c_{\mathrm{T}}^{0}+\bar{K}}{\left\{\left(c_{\mathrm{I}}^{0}+c_{\mathrm{T}}^{0}+\bar{K}\right)^{2}-4 c_{\mathrm{I}}^{0} c_{\mathrm{I}}^{0}\right]^{1 / 2}}-1\right)$

It may be noted that only the definitions of $\bar{K}$ are different for both models. Therefore, in our case the analysis of relaxation amplitudes alone cannot differentiate between the alternative pathways.

\section{4. $\bar{K}$ and reaction enthalpy}

If eq. (8) is inserted into eq. (2), the numerical value of $\bar{K}$ and the absolute value of $\Delta H^{0}$ can be determined. Applying Beer's law to the sum term in eq. (2), we obtain $\sum_{i=1}^{n} \nu_{i}\left(\partial A / \partial c_{i}\right)_{c \neq c_{i}}=\Delta \epsilon d$

where $d$ is the length of light path through the absorbing solution in the observation cell $(c=0.7 \mathrm{~cm})$ and $\Delta \epsilon$ is the difference in the (decadic) extinction coefficients of products and reactants. If eqs. (2) and (8) are now applied to the dissociation directions of scheme (2) and (3), respectively, then $\Delta I^{0}=\Delta I_{\text {diss }}^{0}$. For the case of rapid bimolecular steps and slow structural changes, the overall $\Delta \epsilon$ for both the induced-fit model and the model of selective binding is given by $\Delta \epsilon=$ $\epsilon_{\mathrm{T}}-\epsilon_{\mathrm{IT} \mathrm{T}^{\prime}}$, where it is plausible to assume that $\epsilon_{\mathrm{IT}} \simeq$ $\epsilon_{\mathrm{T}}$ and $\epsilon_{\mathrm{IT}^{\prime}} \simeq \epsilon_{\mathrm{T}^{\prime}}$. Spectrophotometric measurements show that $\epsilon_{\mathrm{I}} \ll \epsilon_{\mathrm{T}}$ and that $\Delta \epsilon=-5.2 \times 10^{-3} \mathrm{M}^{-1} \mathrm{~cm}^{-1}$ for the given experimental conditions. The best fit to the measured relaxation amplitudes as a function of total concentrations is obtained with the parameter values $\bar{K}=5.1( \pm 0.2) \times 10^{-5} \mathrm{M}$ and $\Delta H_{\mathrm{diss}}^{0}=26( \pm 6)$ $\mathrm{kJ} / \mathrm{mole}$. The theoretical functions calculated with these parameter values are included in fig. 3 .

\subsection{Relaxation times}

Once the numerical value for $\bar{K}$ is known, it is convenient to plot the reciprocal values of the relaxation times versus the equilibrium concentrations of the reaction partners [7]. It is this functional dependence between $1 / \tau$ and $\bar{c}_{\mathrm{I}}+\bar{c}_{\mathrm{T}}$ which allows discrimination between the possible alternative reaction pathways depicted in schemes 2 and 3 by simple inspection. Moreover, a closer examination enables us to discuss further modifications of these alternatives. As already mentioned a faster process reflecting simple bimolecular equilibria is not observed. We discuss below that the time constants of bimolecular steps under the given experimental conditions are much smaller than the actually observed relaxation times. Therefore the slow isomerization modes of the limit cases can be described by simple relationships.

The relaxation time of the slow isomerization mode of the induced-fit model is given by [7] :

$\tau^{-1}=k_{2} \frac{\bar{c}_{I}+\bar{c}_{T}}{\bar{c}_{I}+\bar{c}_{T}+K_{1}}+k_{-2}$.

It is readily seen that $\tau^{-1}$ is proportional to the sum of the equilibrium concentrations at low concentrations 
$\left(\bar{c}_{1}+\bar{c}_{\mathrm{T}} \ll K_{1}\right)$ and converges to-the constant values $k_{2}+k_{-2}$, at high excess of the dipeptide. The equilibrium concentrations are in turn connected with the stoichiometric concentrations and to the overall dissociation constant by eq. (7).

On the other hand, the model of selective binding with slow structural transition predicts a concentration dependence of the slow relaxation mode according to $\tau^{-1}=k_{3}+\bar{k}_{-3}\left(\bar{c}_{\mathrm{T}^{\prime}}+K_{4}\right) /\left(\bar{c}_{1}+\bar{c}_{\mathrm{T}^{\prime}}+K_{4}\right)$.

It is sufficient to state that the reciprocal relaxation time of the slow process according to eq. (11), is steadily decreasing with increasing dipeptide concentration; the maximum value is $\tau^{-1}=k_{3}+k_{-3}$ reflecting the intramolecular relaxation of the free protein. This behaviour is already qualitatively in contradiction with the experimental results; $c$ f. fig. 4 . Thus the model of selective binding with rate-determining isomerization is ruled out as the dominant reaction pathway for the observed chemical process.

The mechanistic schemes 2 and 3 also cover the alternative that the bimolecular equilibria are rate-determining, i.e. that the structural isomerizations are very rapid processes. This implies that the intramolecular processes, in order to be fast with respect to the bimolecular ones, should not involve complex (and therefore slow) isomerizations, but rather simple processes. The

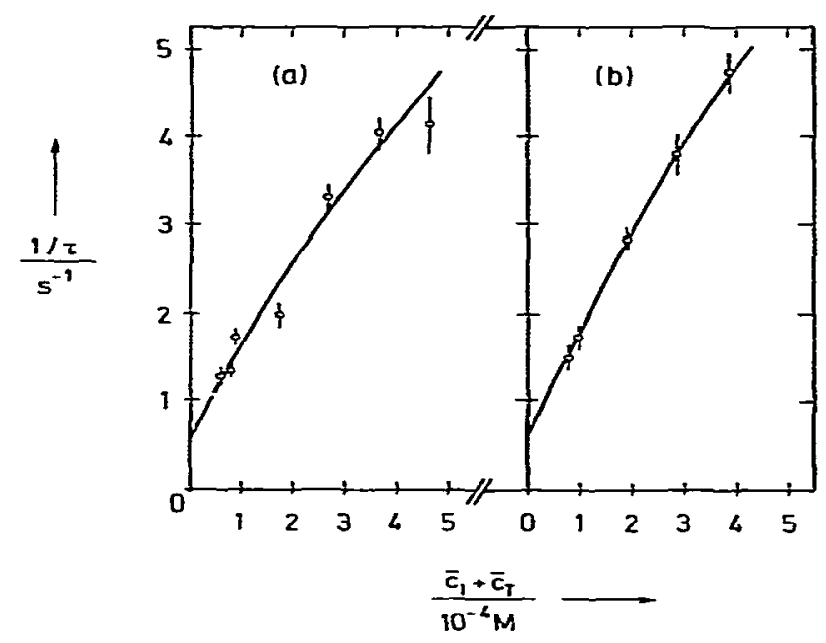

Fig. 4. Reciprocal relaxation time of the slow relaxation mode versus the sum of the equilibrium concentrations of dipeptide and protein with $\bar{K}$-values from table 1 . Theoretical fitting according to eq. (10); (a) $c_{\mathrm{T}}^{0}=5 \times 10^{-5} \mathrm{M}$, (b) $c_{\mathrm{T}}^{0}=2 \times 10^{-5} \mathrm{M}$. theoretical behaviour of the induced-fit model and of the model of selective binding is formally similar under this assumption. For the induced-fit model, the reciprocal values of the time constant of the slow relaxation is strictly linear with the equilibrium concentrations of the reactants:

$\tau^{-1}=k_{1}\left(\bar{c}_{\mathrm{I}}+\bar{c}_{\mathrm{T}}\right) \div k_{-1} /\left(1+K_{2}^{-1}\right)$.

For the model of selective binding, the previous assumptions lead to the expression:

$\tau^{-1}=k_{4}\left\{\bar{c}_{\mathrm{I}} /\left(1+K_{3}\right)+\bar{c}_{\mathrm{T}} / K_{3}\right\}+k_{-4}$.

Since both the kinetic data and the $C D$-results suggest that $K_{3} \gg 1, \tau^{-1}$ is practically linear in $\left(\bar{c}_{1}+\bar{c}_{\mathrm{T}}\right)$, the slope of this relation would then be given by $k_{4} / K_{3}$. However, the experimental reciprocal relaxation times do not fulfill the linear relationship; they rather tend towards a constant value at high concentrations (fig. 4). The inconsistency between the actual data and the predictions for the model of rapid isomerizations may perhaps be seen better from fig. 2 . The plot of $\tau$ versus the ratio $c_{\mathrm{I}}^{0} / c_{\mathrm{T}}^{\mathbf{0}}$ apparently converges to a constant non-zero value; $\tau$ should, however, tend to zero, if the assumption of rate-determining bimolecular equilibria is correct. The analysis of chemical relaxations in terms of total concentrations is straightforward not only for amplitudes but also for time constants. Relationships between equilibrium and total concentrations are given in eqs. (5) and (7).

Among the examined mechanistic schemes, only the conventional induced-fit model is able to correctly reproduce all experimental observations quantitatively. The theoretical functions shown in fig. 4 are calculated for this model by way of least squares' fitting to eq. (10); they are also recalculated and included in fig. 2 . For the two protein concentrations investigated by kinetic titration with the Mle-Val dipeptide, the optimized parameter values [10] for the induced-fit limit case are surnmarized in table 1.

\section{Discussion}

It has been mentioned that the observed chemical relaxation does not appear to reflect rate-limiting bimolecular processes. If protein and dipeptide simply react according to $I+T=I T$, the relaxation time of this reaction would be given by $1 / \tau=k_{1}\left(\bar{c}_{1}+\bar{c}_{\mathrm{T}}+\bar{K}\right)=$ $k_{1}\left[\left(c_{\mathbf{I}}^{0}+c_{\mathrm{T}}^{0}+\bar{K}\right)^{2}-4 c_{\mathrm{I}}^{0} c_{\mathrm{T}}^{0}\right]^{1 / 2}$, see $[6,7]$. Experimental 
Table 1

Kinetic and themodynamic constants of the interaction between pGB-trypsincgen and Ile-Val in $0.1 \mathrm{M}$ Tris $-\mathrm{HCl}$ buffer at $\mathbf{p H}=7.4$ and $296 \mathrm{~K}$, evaluated according to the induced-fit model with rate-limiting intramolecular process.

\section{Sample:}

(a)

\begin{tabular}{lll}
\hline$c_{T}^{0} / \mathrm{M}$ & $5.0 \times 10^{-5}$ & $2.0 \times 10^{-5}$ \\
$\bar{K} / \mathrm{M}$ & $5.1 \times 10^{-5}$ & $5.0 \times 10^{-5}$ \\
$K_{1} / \mathrm{M}$ & $2.0 \times 10^{-3}$ & $2.6 \times 10^{-3}$ \\
$k_{2} / \mathrm{s}^{-1}$ & 21 & 32 \\
$k_{-2} / \mathrm{s}^{-1}$ & 0.57 & 0.64 \\
$\Delta H_{\text {diss }}^{\phi} / \mathrm{kJ} \mathrm{mol}^{-1}$ & 19 & 32 \\
\hline
\end{tabular}

values of $k_{1}$ for diffusion controlled reactions are between $10^{8}$ to $10^{9} \mathrm{M}^{-1} \mathrm{~s}^{-1}$ [7]. With this estimate and the number values for $\bar{K}$ and the total concentrations applied in this study, we calculate $\tau$ to be between 0.1 $\mathrm{ms}$ and $1 \mathrm{~ms}$; two to three orders of magnitude smaller than the actually observed time constants. Furthermore, any process which is bimolecular or bimolecularly ratecontrolled exhibits a strictly linear dependence on the equilibrium concentrations [7]. Thus, both order of magnitude and concentration dependence of the measured relaxation times are inconsistent with a rate-limiting bimolecular reaction. The observed chernical relaxation must therefore reflect a rate-controlling isomerization of the protein in the presence of the dipeptide. Since only one chemical relaxation is observable, it appears that only one pathway in the general interaction scheme 1 is dominantly populated. Within the framework of this scheme quantitative analysis of the concentration dependence of time constants and amplitudes can distinguish between the various alternative mechanisms.

Among these alternatives only the sequential, induced-fit model quantitatively applies to all experimental data. Selective binding to the $\mathbf{T}^{\prime}$-form is not detectable and rapid structural isomerizations coupled to slower, i.e. rate-limiting, bimolecular steps can be ruled out.

In the same concentration range where the results of $\mathrm{CD}$ measurements and of $\mathrm{X}$-ray diffraction analysis have shown that free pGB-trypsinogen has the partially disordered zymogen conformation, whereas in the presence of the dipeptide, the protein-dipeptide complex has a trypsin-like highly ordered structure, a chemical relaxation spectrum is observed. The kinetic data reflect a rate-limiting protein isomerization coupled to a more rapid bimolecular ligand binding. It therefore appears justified to associate the observed slow isomerization with the trypsinogen-trypsin transition. The results of the relaxation kinetic study can then be specifically interpreted (in terms of the induced-fit model): the dipeptide predominantly binds to the partially disordered protein and subsequently induces a structural change to the trypsin-like ordered conformation.

Also, independent of the crystallographic information, the relatively small values of the rate constants for this transition, $k_{2}=26( \pm 6) \mathrm{s}^{-1}$ and $k_{-2}=0.6( \pm 0.04) \mathrm{s}^{-1}$ suggest that larger parts of the protein participate in the structural change. Conformational changes involving only a few molecular bonds have rate constants representative for rotational processes; these however have time constants which are orders of magnitude smaller than the relaxation times observed [7]. Therefore, the isomerization between the trypsinogen conformation and the highly ordered trypsin structure appears to be a whole series of individual conformational steps which cannot yet be resolved with the present relaxation technique. Unfortunately, the thermodynamic data are not informative enough to reveal further details on the protein domain which is structured in the conformational transition. The mean value of the standard reaction enthalpy of the overall process in scheme $2, \Delta H_{\text {diss }}^{\phi}=$ $26( \pm 6) \mathrm{kJ} \mathrm{mol}^{-1}$, probably reflects partial cancellation of positive and negative contributions of a complex process in the macromolecule; the same holds for the overall mean entropy change $\Delta s_{\text {diss }}^{\phi}=4( \pm 20) \mathrm{kJ}^{-1} \mathrm{~mol}^{-1}$ computed from the numerical values of the reaction enthalpy and the overall equilibrium constant. In a similar kinetic study the interaction between a hapten and an immunoglobulin has been investigated [11]. A refined analysis treating separately the respective amplitindes of the fast and the slow normal reaction modes, led to the conclusion that only the full reaction scheme (cf. scheme 1) was able to reproduce all observations correctly. In our case, less ample kinetic information is available; we can observe only one relaxation throughout the whole concentration range. This relaxation process is well reproduced by the induced-fit model up to concentrations where more than $90 \%$ of the protein is present in the ligand-binding form (cf. the theoretical functions in figs. 2-4). If the full reaction scheme 
(scheme 1) with dominant ligand association along the induced-fit pathway at low dipeptide concentrations would hold, the second pathway should come into play particularly at high dipeptide concentrations; and at some dipeptide concentration it should be expected to observe at least two relaxation processes. Ligand binding to the trypsin-like conformation $T$ ' therefore may play only a very minor role, suggesting that $c_{\mathrm{T}^{\prime}}$ is always very small compared to $\varepsilon_{T}$ -

In conclusion, it thus appears that the kinetic constants are consistent with a major structural change involved in the observed chemical relaxation. It should be emphasized that the relaxation kinetic analysis by itself reveals that there is a ligand-induced intramolecular process and that the induced-fit model describes the minimum mechanism; several other mechanisms can be excluded. The kinetic results can then be combined with $C D$ and $X$-ray crystallographic data and the kinetically observed protein isomerization can be specified in terms of the structural transition between a trypsinogen-like and a trypsin-like conformation. The trypsinogen-trypsin system thus represents an instructive example for the complementary character of structural and dynamical information, which together provide a more detailed knowledge of the interaction of the reacting molecules.

\section{Acknowledgements}

We thank Dr. W. Bode for discussion on the crystallographic structure of the trypsinogen-trypsin system. The technical assistance of $R$. Luckinger and $F$. Stocker and the proof reading of the manuscript by Dr. K. Moss is gratefully acknowledged.

\section{References}

[1] H. Fehthammer, W. Bode and R. Huber, J. Mol. Biol. 111 (1977) 415.

[2] W. Bode, J. Mol. Biol. 127 (1979) 357.

[3] M.A. Kerr, K.A. Walsh and H. Neurath, Biochemistry 14 (1975) 5088 .

[4] W. Bode and R. Huber, FEBS Letters 68 (1976) 231.

[5] R. Rigler, C.R. Rabl and T.M. Jovin, Rev. Sci. Instrum. 45 (1974) 580.

[6] R. Winkler-Oswatitsch and M. Eigen, Angew. Chem. 91 (1979) 20.

[7] M. Eigen and L. DeMaeyer, in: Techniques of organic chemistry, Vol. VIII/2, ed. A. Weissberger (J. Wiley, New York, 1963). p. 859.

[8] D.E. Koshland Jr., Proc. Natl. Acad. Sci. USA 44 (1958) 98.

[9] D. Thusius, J. Am. Chem. Soc. 94 (1972) 356.

[10] M.J.D. Powell, subroutine VA04A in Harwell Subroutine Library Atomic Energy Research Establishment, UK.

[11] D. Lancet and I. Pecht, Proc. Natl. Acad. Sci. USA 73 (1976) 3549. 